\title{
Geophysical Research Letters
}

\section{RESEARCH LETTER}

10.1002/2017GL072692

\section{Key Points:}

- First satellite measurement of the ultralow frequency (ULF) wave growth rate

- Measured growth rate consistent with dispersion solver results for observed ion distributions, validating resonant beam instability theory

- Results will inform future missions near shocks and future nonlinear studies related to turbulence and dissipation in the heliosphere

\section{Correspondence to:}

S. Dorfman,

sethd@physics.ucla.edu

\section{Citation:}

Dorfman, S., H. Hietala,

P. Astfalk, and V. Angelopoulos

(2017), Growth rate measurement

of ULF waves in the ion foreshock,

Geophys. Res. Lett., 44, 2120-2128,

doi:10.1002/2017GL072692.

Received 17 JAN 2017

Accepted 18 FEB 2017

Accepted article online 23 FEB 2017

Published online 15 MAR 2017

O2017. American Geophysical Union. All Rights Reserved.

\section{Growth rate measurement of ULF waves in the ion foreshock}

\author{
S. Dorfman ${ }^{1}$, H. Hietala2 ${ }^{D}$, P. Astfalk ${ }^{1,3}$, and V. Angelopoulos ${ }^{2}$ \\ ${ }^{1}$ Department of Physics and Astronomy, University of California, Los Angeles, California, USA, ${ }^{2}$ Department of Earth, \\ Planetary, and Space Sciences, University of California, Los Angeles, California, USA, ${ }^{3}$ Max Planck Institute for Plasma \\ Physics, Greifswald, Germany
}

Abstract We report the first satellite measurement of the ultralow frequency (ULF) wave growth rate in the upstream region of the Earth's bow shock. We employ the two identical ARTEMIS spacecraft orbiting the Moon to characterize crescent-shaped reflected ion beams and relatively monochromatic ULF waves. The event presented here features spacecraft separation of $\sim 2.5$ Earth radii ( $0.9 \pm 0.1$ wavelengths) in the solar wind flow direction along a nearly radial interplanetary magnetic field. The ULF wave growth rate is estimated and found to fall within dispersion solver predictions during the initial growth time. Observed frequencies and wave numbers are also within the predicted range. Other ULF wave properties such as the phase speed, obliquity, and polarization are consistent with expectations from resonant beam instability theory and prior satellite measurements. These results will inform future missions near bow and interplanetary shocks as well as future nonlinear studies related to turbulence and dissipation in the heliosphere.

\section{Introduction}

Waves generated by accelerated particles are important throughout our heliosphere [e.g., Lee et al., 2012]. These particles often gain their energy at shocks via Fermi acceleration [e.g., Jones and Ellison, 1991]. At the Earth's bow shock, this mechanism accelerates ion beams back into the solar wind where they can then generate ultralow frequency (ULF, $1 \mathrm{mHz}$ to $1 \mathrm{~Hz}$ ) waves at a fraction of the ion cyclotron frequency [e.g., Burgess et al., 2012]. These waves are a key component of the diffusive shock acceleration mechanism [Berezhko and Ellison, 1999]. They also influence the shock structure, lead to coherent structures in the magnetosheath, and are a possible source of the ULF waves that play a key role in magnetospheric dynamics [e.g., Wilkinson, 2003; Eastwood et al., 2005a; Omidi et al., 2010].

ULF waves are generated in the upstream region of the Earth's bow shock when an ion beam is accelerated from the shock front back into the solar wind. The interaction of the reflected ions with the background solar wind population may generate the waves via one of the several instability mechanisms. As the beam is initially collimated, the ion-ion right-hand resonant instability and/or the ion-ion right-hand nonresonant instability will be active, depending on beam density [Gary et al., 1981, 1984]. Because the phase speed of the waves generated is much less than the solar wind speed, the waves are then convected by the solar wind back toward the Earth. As the beam loses energy to the waves, beam particles diffuse along curved paths in velocity space; an initially well-collimated beam will evolve into a crescent and then diffuse distribution [Gendrin, 1968]. This decreases the overall growth rate of the instabilities. For diffuse beams, waves may also be generated via an ion-ion left-hand resonant instability [Gary, 1993].

Despite this theoretical understanding of the linear picture, there is at present no definitive observational confirmation of the growth rate in the source region. This is important not only for the local physics but also for understanding the global context involving the nonlinear stage of the waves. Large-amplitude waves convected back toward the shock front may scatter subsequently generated ion beams, modifying the observed distribution [Paschmann et al., 1981; Wang and Lin, 2003]; the waves may also develop into macroscopic entities (foreshock cavitons and short large-amplitude magnetic structures) that affect the shock and magnetosphere [Schwartz et al., 1992; Blanco-Cano et al., 2011]. These large-amplitude waves are also subject to nonlinear interactions [Wang and Lin, 2006; Wang et al., 2015], including daughter wave production via parametric instabilities [Spangler et al., 1997; Narita et al., 2007]. These various nonlinear phenomena may be key to resolving the nature of turbulence and dissipation in our heliosphere. 
Many prior satellite studies have been conducted to observe the properties of ULF waves within $\lesssim 30$ Earth radii $\left(R_{\mathrm{E}}\right)$ distance. lons moving upstream from the bow shock and associated ULF waves were first observed by the Vela satellite in the 1960s [Asbridge et al., 1968; Greenstadt et al., 1968]. Measurements from the two-spacecraft ISEE mission recorded the first detailed measurements of ion beam and wave properties, including phase speed and polarization [Gosling et al., 1978; Hoppe and Russell, 1983]. Sinusoidal waves were observed in conjugation with intermediate crescent-shaped distributions, while more complex steepened structures with multiple frequency peaks appear in concert with diffuse ion beams [Hoppe and Russell, 1983]. More recently, data from the four-spacecraft Cluster mission found the waves to be fast magnetosonic waves [Eastwood et al., 2002] that propagate obliquely to the background magnetic field [Eastwood et al., 2005b] with a distribution of phase speeds centered around the Alfvén speed and resonance condition [Eastwood et al., 2005c; Narita et al., 2004]. Many observed wave properties have also been seen in global hybrid particle in cell simulations of the foreshock [Blanco-Cano et al., 2006, 2009; Strumik et al., 2015]. A recent comparison between near-Earth ULF waves at $X<40 R_{\mathrm{E}}$ and a global hybrid Vlasov code found that the code reproduces the wave properties during a single event in all reported aspects [Palmroth et al., 2015].

These prior studies at $30 R_{E}$ have not measured the ULF wave growth rate, likely due to the proximity of these measurements to the bow shock and the typical spacecraft separation. A shift in the interplanetary magnetic field (IMF) direction will result in a change in direction of the foreshock-generated ion beam. If the observing spacecraft is too close to the Earth, the regions affected by the new and old beams are likely to intersect; this means that waves generated during the previous IMF orientation will interfere with newly generated waves, impeding a clear measurement of the linear growth phase. Furthermore, the typical spacecraft separation in many of these studies is an order of magnitude less than the $\gtrsim R_{\mathrm{E}}$ wavelength of the ULF waves. While ISEE studies include cases with separation up to $1 R_{\mathrm{E}}=6371 \mathrm{~km}$ [Le and Russell, 1990], typical separations of 200 to $2000 \mathrm{~km}$ for ISEE [Hoppe and Russell, 1983] and $600 \mathrm{~km}$ for Cluster [Eastwood et al., 2002, 2005c] are too close for ULF wave growth measurements.

For this reason, the present study makes use of the two Acceleration, Reconnection, Turbulence, and Electrodynamics of the Moon's Interaction with the Sun (ARTEMIS) spacecraft separated by $\approx 1 R_{\mathrm{E}}$ and orbiting the moon $\sim 60 R_{\mathrm{E}}$ from Earth [Angelopoulos, 2011]. When the IMF direction changes such that the foreshock beam reaches ARTEMIS, it is likely that there will be no waves left over from a previous IMF orientation, enabling the first clean measurement of the ULF wave growth rate in the upstream region. Using the fluxgate magnetometer and electrostatic analyzer instruments aboard the two ARTEMIS spacecraft, crescent-shaped ion beams and relatively monochromatic ULF waves are characterized. The event presented here features spacecraft separation in the solar wind flow direction along a nearly radial interplanetary magnetic field. The ULF wave growth rate is estimated and found to fall within dispersion solver predictions during the initial growth time. Observed frequencies and wave numbers are also within the predicted range. Other ULF wave properties such as the phase speed, obliquity, and polarization are consistent with expectations from resonant beam instability theory and prior satellite measurements. Additional features that may be signatures of the nonlinear stages of wave evolution are currently under investigation.

\section{Data, Methods, and Event Overview}

Data from ARTEMIS and other spacecraft plus a new dispersion solver tool are used in the present study. In particular, we use data from the ARTEMIS Fluxgate Magnetometer (FGM) [Auster et al., 2009] to determine growth rate and wave properties as well as background IMF conditions. Distribution function data from the ARTEMIS electrostatic analyzer (ESA) [McFadden et al., 2008a, 2008b] and Solid State Telescope (SST) [Angelopoulos, 2009] is used to determine beam distribution and background plasma properties. Our pristine solar wind measurements are supplemented by ACE and Wind at $\sim 220 R_{\mathrm{E}}$ and Cluster at $\sim 20 R_{\mathrm{E}}$. To compare measured growth rates to theoretical predictions, the new state-of-the-art LEOPARD (Linear Electromagnetic Oscillations in Plasmas with Arbitrary Rotationally-symmetric Distributions) solver is employed with gyroaveraged ARTEMIS ion distributions [Astfalk and Jenko, 2017].

The geometry of our event is shown in Figure 1 (left). The IMF is nearly radial, as indicated by the green magnetic field lines measured at each spacecraft. The two ARTEMIS spacecraft P1 and P2 are shown, separated by $\sim 2.5 R_{\mathrm{E}}$ in $x$ and $0.55 \pm 0.05 R_{\mathrm{E}}$ in the perpendicular direction (including $y$ (shown) and $z$ (not shown)). The latter distance is well within the $\sim 1 R_{\mathrm{E}}$ estimate over which Le and Russell [1990] determined that ULF waves remain correlated perpendicular to the solar wind flow in ISEE measurements as well as the $\sim 3 R_{\mathrm{E}}$ over which Archer et al. [2005] found that the waves remain planar in the perpendicular direction in Cluster data. We therefore 




Figure 1. Event geometry. (left) Position of the ARTEMIS spacecraft P1 and P2 in the $X Y$ plane in geocentric solar ecliptic (GSE) coordinates [Kivelson and Russell, 1995]. The Earth and bow shock are on the left; in the upper right is a small box indicating the position of the moon and the two orbiting ARTEMIS spacecraft. Magnetic field orientation measured at each spacecraft is indicated by green lines, and the measured solar wind direction is given by a gray arrow. Both are averaged over the time period in the figure title. (right) Cartoon showing two wave packets, $w_{A}$ and $w_{B}$, that convect and grow. Positions of the upstream and downstream spacecraft are indicated.

expect that the solar wind, which is in the $-x$ direction, will convect waves from the upstream spacecraft (P1) to the downstream spacecraft (P2) along the nearly radial magnetic field. Based on data from the ACE and Wind spacecraft located far upstream at $\sim 220 R_{E}$, we estimate the pristine solar wind $x$ speed to be $365 \pm 5 \mathrm{~km} / \mathrm{s}$, the proton temperature as $6.5 \pm 2.5 \mathrm{eV}$, and the ion density as $4.2 \pm 0.7 / \mathrm{cm}^{3}$.

A cartoon of how these ULF waves are expected to convect and grow under radial IMF conditions is shown in Figure 1 (right). At time $t_{0}$, ULF wave packet $w_{A}$ is generated at the upstream (us) spacecraft by an ion beam traveling away from Earth. As time advances, the plasma parcel containing $w_{A}$ (represented by a 3-D box) is convected downstream by the solar wind $\left(v_{\mathrm{sw}}\right)$ and passes the location of the downstream (ds) spacecraft at $t_{1}$. Meanwhile, $w_{A}$ continues to gain energy from the ion beam and grows in amplitude. In the solar wind rest frame, $w_{A}$ is moving at a phase speed $v_{\mathrm{ph}} \sim v_{\text {Alfvén }} \ll v_{\mathrm{sw}}$ in the same direction as the beam; therefore, $w_{A}$ moves to the right within the plasma parcel. Similar behavior is seen for a second wave $w_{B}$. Resonant beam instability theory predicts that the waves will be intrinsically right-hand polarized in the solar wind frame [Gary, 1993], but because of the backward convection by the solar wind, the waves will have apparent left-hand polarization in the spacecraft rest frame. To compare observations of $w_{A}$ on the upstream and downstream spacecraft, data from the upstream spacecraft must be shifted forward by the appropriate amount; this is done in section 4.

Figure 2 displays data observed by the two ARTEMIS spacecraft during the event showcased in Figure 1. Shortly after a jump in IMF $B_{z}$ (red curve in Figure $2 d$ ) at about 19:13 UT, an ion beam is observed starting at 19:16 UT (Figures 2a, 2c, and 2f) followed by the onset of ULF waves at 19:21 UT (Figure 2d). At the three times indicated by the vertical dashed lines, the ion distribution functions in Figures $2 g-2 i$ show both the solar wind core distribution and the ion beam. Note that the ion electrostatic analyzer (ESA) instrument [McFadden et al., 2008a, 2008b] which recorded this data does not have sufficient resolution to resolve the core temperature; furthermore, the extension on the left side of the core represents solar wind alpha particles assumed to be protons by ESA. These distributions are processed by subtracting the one-count level and then averaging over gyroangle in each energy bin. This processing method is chosen so that these distributions may be fed directly into LEOPARD and the results compared to the ULF wave growth rate measured from the time traces in Figure 2.

\section{Theory: Resonant Ion-Ion Beam Instabilities}

The ULF waves are produced via a Doppler shifted anomalous cyclotron resonance. lons gyrate in a left-hand sense and therefore require a left-hand polarized electric field at a frequency that matches their gyromotion in order to undergo resonance. Therefore, in the rest frame of the beam ions, the waves must be left-hand polarized with frequency $n \Omega_{i}$, where $n$ is an integer. However, this condition can be satisfied for waves with both left- and right-handed "intrinsic polarization," defined as the polarization in the solar wind rest frame. The Doppler shift between the beam and solar wind frames is described by the equation [Gary, 1993]

$$
\omega-k_{\|} v_{\text {beam }}= \pm \Omega_{i}
$$



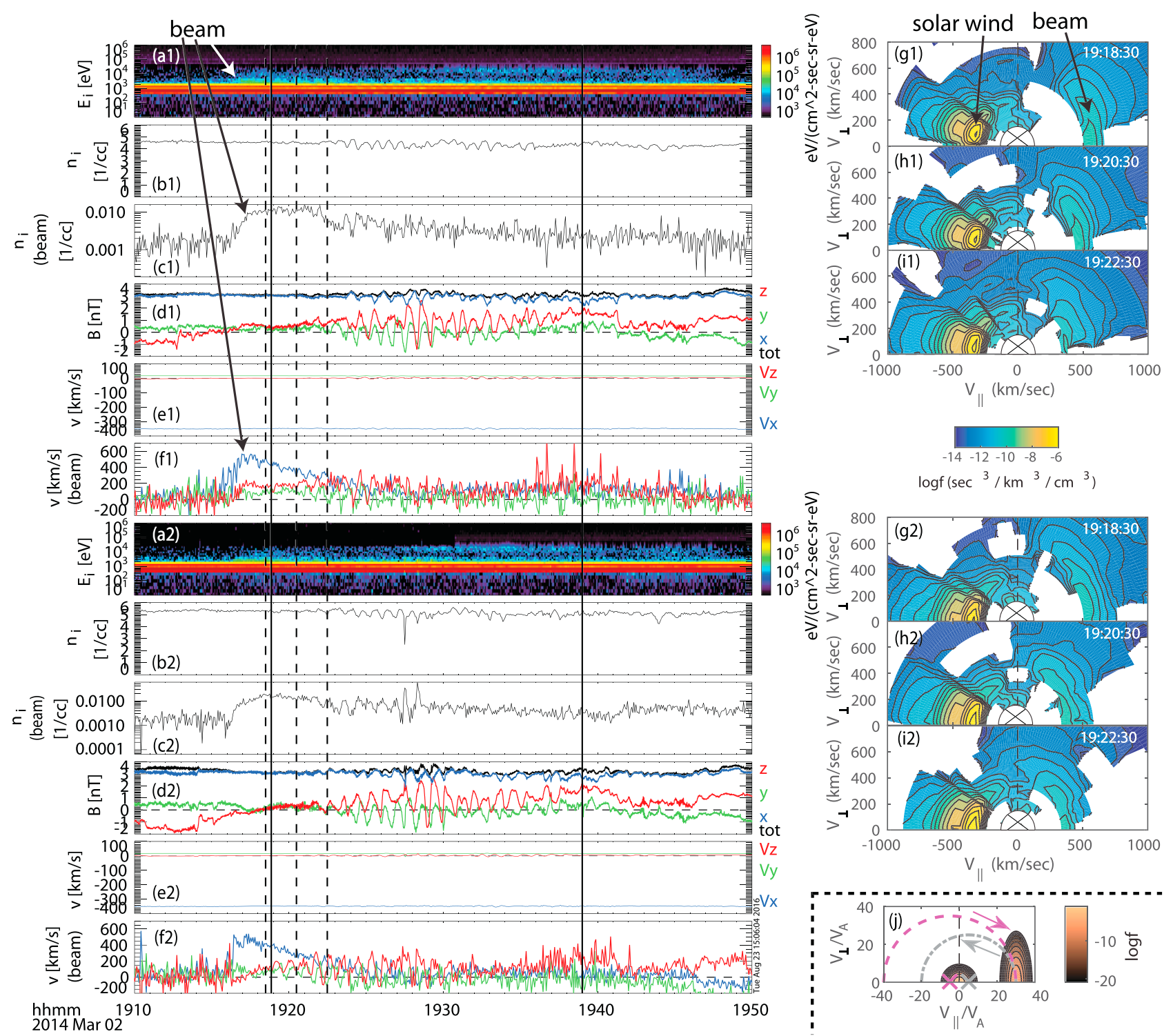

Figure 2. Event summary plot. Plotted against time for each spacecraft $(1=\mathrm{P} 1 ; 2=\mathrm{P} 2)$ are (a) combined energy flux spectrum from the electrostatic analyzer (ESA) and Solid State Telescope (SST). (b) lon density from reduced ion distribution functions. (c) Beam density including particles greater than 50 eV within $120^{\circ}$ of GSE $x$. (d) Magnetic field from the fluxgate magnetometer (FGM). (e) Bulk ion velocity from onboard moments. (f) Beam velocity with the beam defined as in Figure $2 \mathrm{c}$. The time between the two solid vertical lines is the time of interest for wave data used in subsequent figures. ( $g$-i) Distribution function plots taken at the times indicated by the three vertical dashed lines in Figures $2 a-2 f$ and averaged over gyroangle. Thirty second intervals are used centered at the times shown. (j) A model distribution with example diffusion paths; arrows indicate the direction for the loss of energy to waves. 
Anomalous cyclotron resonance occurs when an intrinsically right-hand-polarized wave is overtaken by a beam traveling in the same direction $\left(k_{\|} v_{\text {beam }}>0\right)$ in the solar wind frame; the wave is then seen as left-handed in the beam frame due to the Doppler shift.

For waves to grow on resonance, they must gain energy from particles. In the frame moving with the wave, time derivatives vanish; therefore, Faraday's law predicts no electromagnetic electric field in this frame. Since a wave with magnetic field alone cannot change particle energy, particle diffusion paths in velocity space must conserve energy in the frame comoving with the wave. This means that in the solar wind frame, these paths take the form of a circle centered at $\omega / k_{\|}$, as shown in Figure $2 \mathrm{j}$ [Kennel and Petschek, 1966; Gendrin, 1968]. Because the distribution function shown in the figure is peaked around $v_{\|}=v_{\text {beam }}$, only diffusion paths centered at a positive wave parallel phase speed in the solar wind frame will result in beam particles moving down a gradient in velocity space to lose energy to the wave. Therefore, only anomalous cyclotron resonance is predicted for the beam distribution in Figure $2 \mathrm{j}$. This produces intrinsically right-hand circularly polarized waves and is known as the ion-ion right-hand resonant instability. While a nonresonant instability is also possible if the beam speed is large enough [Gary et al., 1981, 1984], this process is not predicted for the event in Figure 2.

\section{Results}

\subsection{Growth Rate and Obliquity}

The growth rate of the observed ULF waves is successfully measured in Figure 3. A wave observed on the upstream (us) spacecraft is observed about $45 \mathrm{~s}$ later on the downstream (ds) spacecraft. Therefore, to align the waves in Figure 3a, the red time axis for upstream spacecraft data is shifted forward $45 \mathrm{~s}$ with respect to the blue time axis for downstream data. From the wave field amplitude displayed in Figure 3b, we clearly identify the time the wave is growing and indicate this growth with a dashed line. This gives a growth rate estimate of $0.0079 / \mathrm{s}$; however, the ion distributions that generate the waves (Figures $2 \mathrm{~g}-2 \mathrm{i}$ ) evolve quickly compared to this growth time. Therefore, this estimate represents different waves as they pass by the spacecraft. A more accurate measure of the growth rate requires the simultaneous two-spacecraft measurement displayed in Figure 3c. The ratio of downstream to upstream amplitudes shown represents the growth of a single wave as it is convected by the solar wind from the upstream to the downstream spacecraft. Within the gray shaded region at the center of the growth period, a 1.57 amplitude ratio leads to an estimated growth rate of 0.010/s. Using a magnetic field magnitude of $3 \mathrm{nT}$, the normalized growth rate is estimated to be $\gamma / \Omega_{i} \sim 0.035$.

The waves are measured to be oblique in Figure $3 \mathrm{~d}$ using standard minimum variance techniques and error analysis [Sonnerup and Scheible, 1998; Mazelle et al., 2003]. This is consistent with Strumik et al. [2015]: even though parallel waves have the highest growth rates, a larger solid angle $k$ space is available for oblique waves to grow. Furthermore, from 19:23 UT to 19:29 UT, the waves become more oblique as their amplitude increases, consistent with work that suggests that changes in the beam properties are responsible [Palmroth et al., 2015]. Oblique waves may also be generated by beam ring distributions, but unlike the waves observed in Figure 2, such waves are highly compressive [Blanco-Cano et al., 2006].

\subsection{Phase Speed and Polarization}

The measured phase speed and polarization of the ULF waves is broadly consistent with activity of the ion-ion right-hand resonant instability. Waves produced by this instability will have intrinsic right-hand polarization but will appear left-hand circularly polarized in the spacecraft frame where the beam overtakes the waves. Consistent with this, most of the wave power observed in Figures $3 \mathrm{e}-3 \mathrm{~h}$ is left-hand circularly polarized. Two main peaks are observed at 0.0167 and $0.0198 \mathrm{~Hz}$. Within the initial growth time (vertical gray shaded region), Figure $3 \mathrm{~h}$ shows that only apparent left-hand modes grow as they convect from the upstream to the downstream spacecraft. At the same time, Figure 3i shows that the apparent left-hand wave phase speed is $\sim 330 \mathrm{~km} / \mathrm{s}$ in the spacecraft frame which is slower than the solar wind speed of $365 \pm 5 \mathrm{~km} / \mathrm{s}$ by about an Alfvén speed $\left(v_{A} \sim 32 \mathrm{~km} / \mathrm{s}\right)$, within the range of phase speeds expected for the instability. These numbers imply that the spacecraft separation of $\sim 2.5 R_{\mathrm{E}}$ corresponds to $0.9 \pm 0.1$ wavelengths, confirming that there is sufficient room for the ULF waves to grow as they convect between the two spacecraft.

Since the calculated phase speed in the solar wind frame is about one tenth of the spacecraft frame value, the observed wave frequencies will be about 10 times lower when shifted to the solar wind frame; the two peaks observed will occur at $\sim 0.040 f_{c i}$ and $\sim 0.045 f_{c i}$ in this intrinsic frame. At these low values of $f / f_{c i}$ we expect the phase and group speeds to be nearly identical [Cramer, 2011]; consistent with this, both the wave envelope and phases align for the time-shifted data in Figures $3 \mathrm{a}, 3 \mathrm{f}$, and $3 \mathrm{~g}$. 

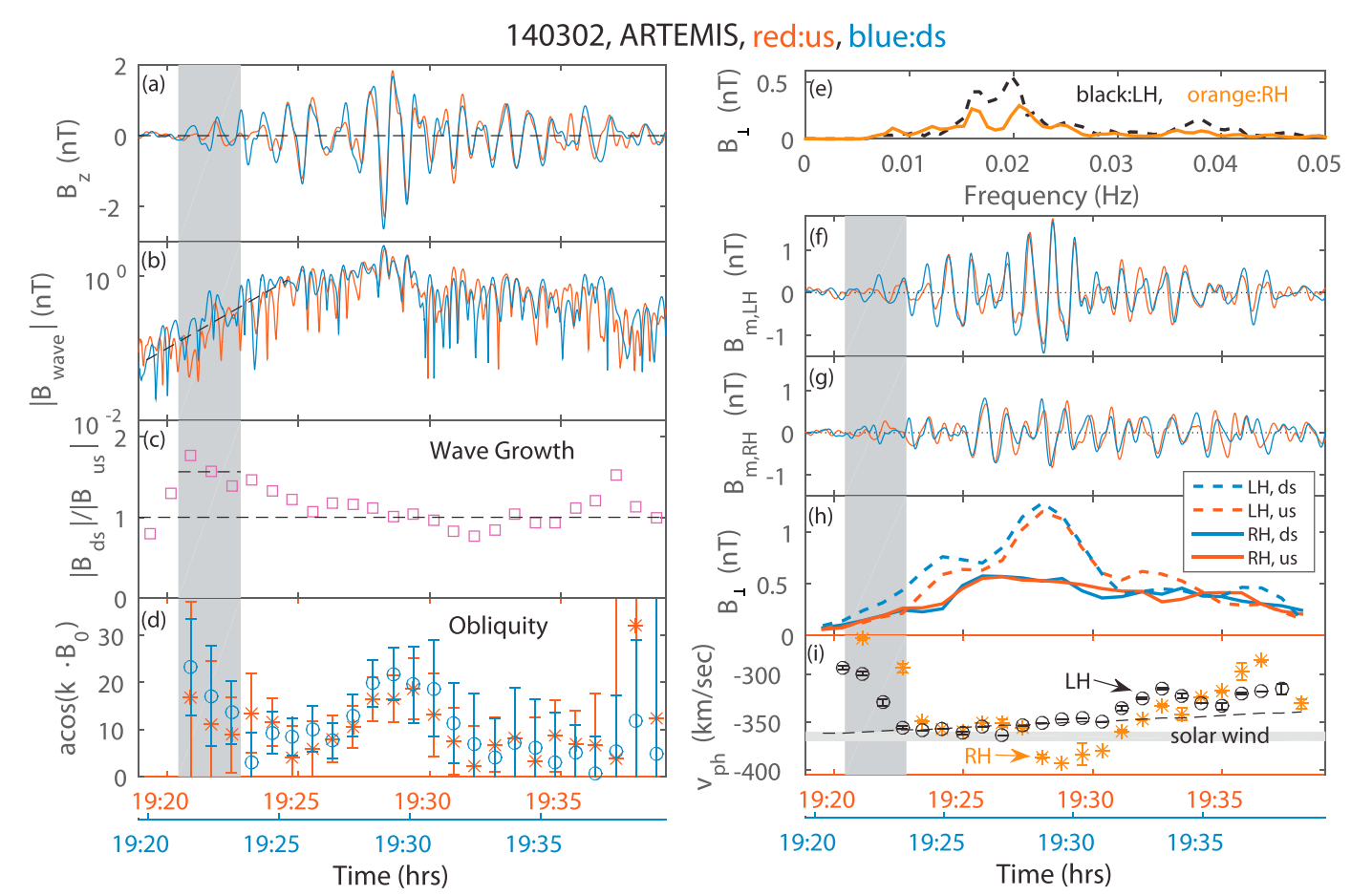

Figure 3. Growth rate measurement of ULF waves and phase velocity and polarization measurements. Downstream spacecraft (ARTEMIS P2) in blue and upstream spacecraft (ARTEMIS P1) in red. Note that the time axes used for the two spacecraft (bottom) are offset from each other by $45 \mathrm{~s}$. Time traces are filtered between $0.007 \mathrm{and} 0.05 \mathrm{~Hz}$. (a) Filtered $z$ component of the fluctuating magnetic field signal. (b) Amplitude of the fluctuating magnetic field in the same frequency range. The two field components perpendicular to the minimum variance direction are used. (c) Ratio of amplitude between the downstream and upstream spacecraft. Amplitude is as defined in Figure $3 \mathrm{~b}$ and averaged over $143 \mathrm{~s}$ time intervals, the length of the gray shaded region. (d) Obliquity calculated from minimum variance analysis averaged over the same time intervals. (e) Magnetic field spectrum perpendicular to the minimum variance direction averaged over the two spacecraft. Fluctuations are decomposed into apparent left-hand (black dashed) and right-hand (orange solid) circularly polarized components in the spacecraft frame. The entire time window shown in the other panels is used. $(\mathrm{f}, \mathrm{g})$ The components perpendicular to the minimum variance direction are decomposed into apparent left-hand and right-hand components. The $m$ direction associated with the intermediate eigenvalue in minimum variance coordinates is shown for both spacecraft. (h) Power in apparent left-hand and right-hand modes averaged over $143 \mathrm{~s}$ intervals. (i) Phase speed measurement in the spacecraft frame for apparent left-hand (black circles) and right-hand (orange stars) components averaged over the same $143 \mathrm{~s}$ intervals. Phase speed is determined by dividing the spacecraft distance in $x$ by the time delay between signals. The dashed black line is the phase speed measured at each time assuming a delay of $45 \mathrm{~s}$ between spacecraft; the slope is due to the spacecraft moving closer together.

Figure 3 also shows significant power in waves that have apparent right-hand polarization ("right-hand waves"), the origin of which is still under investigation. Unlike the waves with apparent left-hand polarization ("left-hand waves"), Figure $3 \mathrm{~h}$ shows that the right-hand waves retain the same amplitude as they convect between the two spacecraft. This suggests that the right-hand waves do not grow in the observation region but could instead be produced further upstream and then convected to the observation region by the solar wind. However, they are unlikely to be background solar wind fluctuations: since the right-hand wave amplitude increases starting at 19:24 UT, just as the left-hand waves reach an amplitude plateau in time, it seems plausible that the production of right-hand waves could be related to an amplitude threshold in left-hand wave power. Comparing Figures $3 \mathrm{f}$ and $3 \mathrm{~g}$, bursts of right-hand waves tend to appear just after bursts of left-hand waves. Furthermore, as the left-hand waves reach maximum amplitude at 19:28 UT in Figure 3h, the right-hand waves overtake the solar wind by about an Alfvén speed in Figure 3i. At the same time in Figure 3g, the red trace leads the blue trace, confirming the observed change in phase speed. This means that these waves at 19:28 UT propagate toward Earth in the solar wind frame and are therefore both apparently and intrinsically right-hand polarized. Because the beam is moving away from Earth in this frame, $k_{\|} v_{\text {beam }}<0$. As discussed in section 3 , this means that anomalous cyclotron resonance is not possible and these 


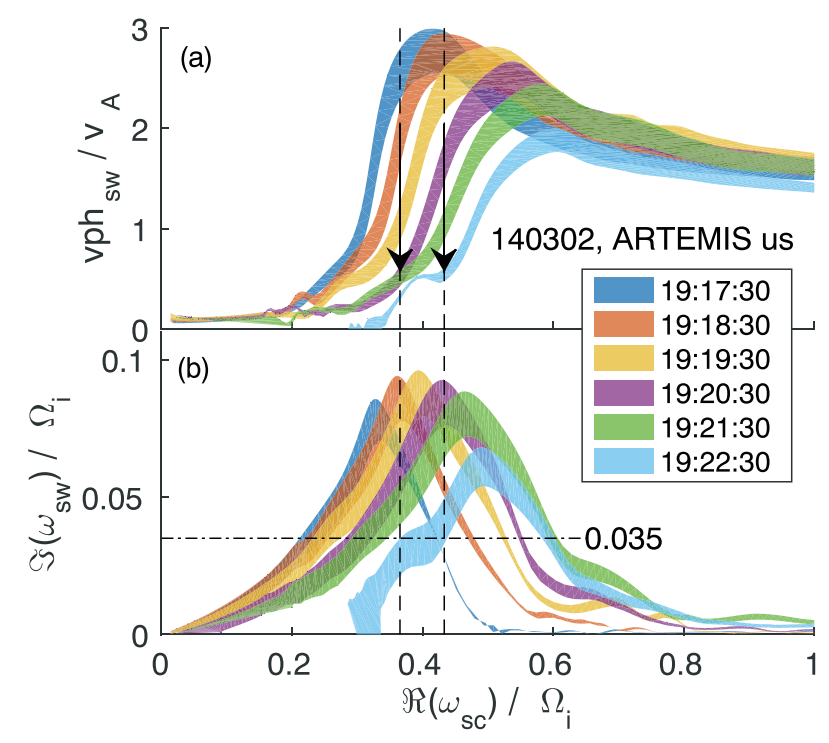

Figure 4. Wave properties based on distribution functions measured at the upstream spacecraft. (a) Phase speed and (b) growth rate in the solar wind frame are plotted against the real part of the frequency in the spacecraft frame for six times during the event. Thirty second intervals are used centered at the times shown. The finite width of each curve is due to the uncertainty in background solar wind density $4.5 \pm 1.0 / \mathrm{cm}^{3}$. Vertical dashed lines are the frequencies of the two peaks measured in Figure $3 e$. waves with apparent right-hand polarization observed at 19:28 UT are not due to the ion-ion right-hand resonant instability. An intriguing alternative source for these waves is a parametric instability of the beam-produced waves in the solar wind frame; this could produce backward propagating ULF waves with the same intrinsic right-hand polarization.

\section{Discussion: Comparison With Theory}

Wave growth rates predicted from the measured distributions are in line with spacecraft measurements. This may be seen by plugging the distributions at the top right of Figure 2 into the LEOPARD solver. This is done using actual ARTEMIS beam distributions with a model Maxwellian core; the core density and temperature are selected by using Wind, ACE, and Cluster to complement low-resolution ARTEMIS data. The resulting phase speeds and growth rates are shown in Figure 4. The finite width of each curve is due to uncertainty in the core density, $4.5 \pm 1.0 / \mathrm{cm}^{3}$; the results are comparatively insensitive to core temperature. Notice that the dominant frequencies observed in the spacecraft frame in Figure $3 e$ (indicated by the vertical dashed lines in Figure 4) fall amid the peaks in the calculated growth rate curves in Figure $4 \mathrm{~b}$. Furthermore, our growth rate estimate of $\gamma / \Omega_{i} \sim 0.035$ at 19:21:30 UT is the location where the 19:22:30 UT growth rate curve crosses the vertical dashed line. Because the growth rate curves evolve surprisingly quickly (over a timescale of $60 \mathrm{~s}$ ), but the ARTEMIS wave growth estimate in Figure $3 \mathrm{c}$ is averaged over $143 \mathrm{~s}$, we can conclude that our estimate is within range, but it is difficult to be more precise. Even though the growth rate curve at 19:22:30 UT peaks to the right of the dashed frequencies, because the growth of these frequencies was favored at an earlier time, they likely continue to grow even after the ion distribution function changes.

Figure 4 implies that the dynamic nature of the foreshock may play a key role in the observed ULF wave properties. Consider a wave generated at a fixed spacecraft frame frequency ( $x$ axis) in Figure $4 a$. As time advances, the phase speed ( $y$ axis) at the frequency of one of the vertical dashed lines will decrease, following the two black arrows. This trend is consistent with Figure $3 i$ which shows a similar decrease in the wave phase speed of the left-hand component in the initial growth period. (Note that zero phase speed in the solar wind frame in Figure $4 \mathrm{a}$ corresponds to the ULF waves traveling at the solar wind speed in the spacecraft frame in Figure 3i.)

\section{Conclusions}

In this letter, we have reported the first satellite measurement of the ULF wave growth rate in the upstream region. Using the fluxgate magnetometer and electrostatic analyzer instruments aboard the two ARTEMIS spacecraft, crescent-shaped ion beams and relatively monochromatic ULF waves are characterized. The event presented here features spacecraft separation of $\sim 2.5 R_{E}(0.9 \pm 0.1$ wavelengths) in the solar wind flow direction along a nearly radial interplanetary magnetic field. The ULF wave growth rate is estimated as $\gamma / \Omega_{i} \sim 0.035$ and found to fall within dispersion solver predictions during the initial growth time. Observed frequencies and wave numbers are also within the predicted range. Other ULF wave properties such as the phase speed, obliquity, and polarization are consistent with expectations from theory and prior satellite measurements. 
These results shed insight into the linear properties of ULF waves that will be useful in other contexts. For example, ULF waves are observed at various planetary bow shocks [Hoppe and Russell, 1982] as well as interplanetary shocks [Kajdič et al., 2012; Blanco-Cano et al., 2016]; while these observations typically feature only a single spacecraft, the theory-observation comparison presented in the present paper suggests that dispersion solver results could provide a reasonable estimate of ULF wave growth for missions with well-resolved ion distributions. Such an estimate may be useful for the Magnetospheric Multiscale (MMS) mission as well as the upcoming Solar Probe Plus and Solar Orbiter missions. In winter 2017-2018 MMS will sweep across the dayside at $\sim 25 R_{\mathrm{E}}$, and ULF waves are expected to be frequently observed. Furthermore, our results can inform simulations of particle acceleration at shocks in the heliosphere and beyond.

The present measurements of the linear wave properties will also enable future studies of the nonlinear evolution. Right-hand wave power observed during the event studied is a prime candidate for future studies of parametric processes. The surprisingly fast evolution of the ion distribution merits further investigation that could quasi-linearly explain the two frequency peaks observed. Additional events characterized by diffuse ion beams are also under investigation. These investigations will help us understand prior nonlinear observations [Schwartz et al., 1992; Spangler et al., 1997; Blanco-Cano et al., 2011; Wang et al., 2015], providing the building blocks for future studies of turbulence and dissipation in the heliosphere, a focus of both the Turbulence Heating ObserveR (THOR) mission in development and the extended MMS mission.

\section{Acknowledgments}

ARTEMIS data used in this work were obtained from the ARTEMIS website http://themis.ssl.berkeley.edu; ACE/Wind data were obtained from http://cdaweb.sci.gsfc.nasa.gov/. The THEMIS/ARTEMIS project is funded through NASA grant NAS5-02099. S. Dorfman was supported by a NASA Jack Eddy Postdoctoral Fellowship and acknowledges fruitful discussions with Xin An and Marty Lee. H. Hietala acknowledges fruitful discussions within the international team Jets Downstream of Collisionless Shocks at the International Space Science Institute (ISSI) in Bern

\section{References}

Angelopoulos, V. (2009), The THEMIS mission, in The THEMIS Mission, edited by J. L. Burch and V. Angelopoulos, pp. 5-34, Springer, New York, doi:10.1007/978-0-387-89820-9_2.

Angelopoulos, V. (2011), The ARTEMIS mission, Space Sci. Rev., 165(1), 3-25, doi:10.1007/s11214-010-9687-2.

Archer, M., T. S. Horbury, E. A. Lucek, C. Mazelle, A. Balogh, and I. Dandouras (2005), Size and shape of ULF waves in the terrestrial foreshock, J. Geophys. Res., 110, A05208, doi:10.1029/2004JA010791.

Asbridge, J. R., S. J. Bame, and I. B. Strong (1968), Outward flow of protons from the Earth's bow shock, J. Geophys. Res., 73(17), 5777-5782, doi:10.1029/JA073i017p05777.

Astfalk, P., and F. Jenko (2017), Leopard: A grid-based dispersion relation solver for arbitrary gyrotropic distributions, J. Geophys. Res. Space Physics, 122, 89-101, doi:10.1002/2016JA023522.

Auster, H. U., et al. (2009), The THEMIS fluxgate magnetometer, in The THEMIS Mission, edited by J. L. Burch and V. Angelopoulos, pp. 235-264, Springer, New York, doi:10.1007/978-0-387-89820-9_11.

Berezhko, E. G., and D. C. Ellison (1999), A simple model of nonlinear diffusive shock acceleration, Astrophys. J., 526(1), $385-399$.

Blanco-Cano, X., N. Omidi, and C. T. Russell (2006), Macrostructure of collisionless bow shocks: 2. ULF waves in the foreshock and magnetosheath, J. Geophys. Res., 111, A10205, doi:10.1029/2005JA011421.

Blanco-Cano, X., N. Omidi, and C. T. Russell (2009), Global hybrid simulations: Foreshock waves and cavitons under radial interplanetary magnetic field geometry, J. Geophys. Res., 114, A01216, doi:10.1029/2008JA013406.

Blanco-Cano, X., P. Kajdič, N. Omidi, and C. T. Russell (2011), Foreshock cavitons for different interplanetary magnetic field geometries: Simulations and observations, J. Geophys. Res., 116, A09101, doi:10.1029/2010JA016413.

Blanco-Cano, X., P. Kajdič, E. Aguilar-Rodríguez, C. T. Russell, L. K. Jian, and J. G. Luhmann (2016), Interplanetary shocks and foreshocks observed by stereo during 2007-2010, J. Geophys. Res. Space Physics, 121, 992-1008, doi:10.1002/2015JA021645.

Burgess, D., E. Möbius, and M. Scholer (2012), Ion acceleration at the Earth's bow shock, Space Sci. Rev., 173(1), 5-47, doi:10.1007/s11214-012-9901-5.

Cramer, N. F. (2011), The Physics of Alfvén Waves, Wiley-VCH, Berlin.

Eastwood, J. P., A. Balogh, M. W. Dunlop, T. S. Horbury, and I. Dandouras (2002), Cluster observations of fast magnetosonic waves in the terrestrial foreshock, Geophys. Res. Lett., 29(22), 2046, doi:10.1029/2002GL015582.

Eastwood, J. P., E. A. Lucek, C. Mazelle, K. Meziane, Y. Narita, J. Pickett, and R. A. Treumann (2005a), The foreshock, Space Sci. Rev., 118(1), 41-94, doi:10.1007/s11214-005-3824-3.

Eastwood, J. P., A. Balogh, E. A. Lucek, C. Mazelle, and I. Dandouras (2005b), Quasi-monochromatic ULF foreshock waves as observed by the four-spacecraft Cluster mission: 2. Oblique propagation, J. Geophys. Res., 110, A11220, doi:10.1029/2004JA010618.

Eastwood, J. P., A. Balogh, E. A. Lucek, C. Mazelle, and I. Dandouras (2005c), Quasi-monochromatic ULF foreshock waves as observed by the four-spacecraft Cluster mission: 1. Statistical properties, J. Geophys. Res., 110, A11219, doi:10.1029/2004JA010617.

Gary, S. (1993), Theory of Space Plasma Microinstabilities, Cambridge Univ. Press, Cambridge, U. K.

Gary, S. P., J. T. Gosling, and D. W. Forslund (1981), The electromagnetic ion beam instability upstream of the Earth's bow shock, J. Geophys. Res., 86(A8), 6691-6696, doi:10.1029/JA086iA08p06691.

Gary, S. P., C. W. Smith, M. A. Lee, M. L. Goldstein, and D. W. Forslund (1984), Electromagnetic ion beam instabilities, Phys. Fluids, 27(7), $1852-1862$, doi:10.1063/1.864797.

Gendrin, R. (1968), Pitch angle diffusion of low energy protons due to gyroresonant interaction with hydromagnetic waves, J. Atmos. Terr. Phys., 30(7), 1313-1330, doi:10.1016/S0021-9169(68)91158-6.

Gosling, J. T., J. R. Asbridge, S. J. Bame, G. Paschmann, and N. Sckopke (1978), Observations of two distinct populations of bow shock ions in the upstream solar wind, Geophys. Res. Lett., 5(11), 957-960, doi:10.1029/GL005i011 p00957.

Greenstadt, E. W., I. M. Green, G. T. Inouye, A. J. Hundhausen, S. J. Bame, and I. B. Strong (1968), Correlated magnetic field and plasma observations of the Earth's bow shock, J. Geophys. Res., 73(1), 51-60, doi:10.1029/JA073i001p00051.

Hoppe, M. M., and C. T. Russell (1982), Particle acceleration at planetary bow shock waves, Nature, 295(5844), 41-42, doi:10.1038/295041a0.

Hoppe, M. M., and C. T. Russell (1983), Plasma rest frame frequencies and polarizations of the low-frequency upstream waves: ISEE 1 and 2 observations, J. Geophys. Res., 88(A3), 2021-2027, doi:10.1029/JA088iA03p02021.

Jones, F. C., and D. C. Ellison (1991), The plasma physics of shock acceleration, Space Sci. Rev., 58(1), 259-346, doi:10.1007/BF01206003. 
Kajdič, P., X. Blanco-Cano, E. Aguilar-Rodriguez, C. T. Russell, L. K. Jian, and J. G. Luhmann (2012), Waves upstream and downstream of interplanetary shocks driven by coronal mass ejections, J. Geophys. Res., 117, A06103, doi:10.1029/2011JA017381.

Kennel, C. F., and H. E. Petschek (1966), Limit on stably trapped particle fluxes, J. Geophys. Res., 71(1), 1-28, doi:10.1029/JZ071i001 p00001.

Kivelson, M., and C. Russell (1995), Introduction to Space Physics, Cambridge Atmos. and Space Sci. Ser., Cambridge Univ. Press, Cambridge, U. K.

Le, G., and C. T. Russell (1990), A study of the coherence length of ULF waves in the Earth's foreshock, J. Geophys. Res., 95(A7), 10,703-10,706, doi:10.1029/JA095iA07p10703.

Lee, M. A., R. A. Mewaldt, and J. Giacalone (2012), Shock acceleration of ions in the heliosphere, Space Sci. Rev., 173(1), 247-281, doi:10.1007/s11214-012-9932-y.

Mazelle, C., et al. (2003), Production of gyrating ions from nonlinear wave-particle interaction upstream from the Earth's bow shock: A case study from Cluster-CIS, Planet. Space Sci., 51(12), 785-795, doi:10.1016/j.pss.2003.05.002. key Problems in Space Physics: Thin Magnetospheric Boundaries.

McFadden, J. P., C. W. Carlson, D. Larson, J. Bonnell, F. Mozer, V. Angelopoulos, K.-H. Glassmeier, and U. Auster (2008a), THEMIS ESA first science results and performance issues, Space Sci. Rev., 141(1), 477-508, doi:10.1007/s11214-008-9433-1.

McFadden, J. P., C. W. Carlson, D. Larson, M. Ludlam, R. Abiad, B. Elliott, P. Turin, M. Marckwordt, and V. Angelopoulos (2008b), The THEMIS ESA plasma instrument and in-flight calibration, Space Sci. Rev., 141(1), 277-302, doi:10.1007/s11214-008-9440-2.

Narita, Y., et al. (2004), Alfvén waves in the foreshock propagating upstream in the plasma rest frame: Statistics from cluster observations, Ann. Geophys., 22(7), 2315-2323, doi:10.5194/angeo-22-2315-2004.

Narita, Y., K. H. Glassmeier, M. Fränz, Y. Nariyuki, and T. Hada (2007), Observations of linear and nonlinear processes in the foreshock wave evolution, Nonlinear Processes Geophys., 14(4), 361-371, doi:10.5194/npg-14-361-2007.

Omidi, N., J. P. Eastwood, and D. G. Sibeck (2010), Foreshock bubbles and their global magnetospheric impacts, J. Geophys. Res., 115, A06204, doi:10.1029/2009JA014828.

Palmroth, M., et al. (2015), ULF foreshock under radial IMF: THEMIS observations and global kinetic simulation Vlasiator results compared, J. Geophys. Res. Space Physics, 120, 8782-8798, doi:10.1002/2015JA021526.

Paschmann, G., N. Sckopke, I. Papamastorakis, J. R. Asbridge, S. J. Bame, and J. T. Gosling (1981), Characteristics of reflected and diffuse ions upstream from the Earth's bow shock, J. Geophys. Res., 86(A6), 4355-4364, doi:10.1029/JA086iA06p04355.

Schwartz, S. J., D. Burgess, W. P. Wilkinson, R. L. Kessel, M. Dunlop, and H. Lühr (1992), Observations of short large-amplitude magnetic structures at a quasi-parallel shock, J. Geophys. Res., 97(A4), 4209-4227, doi:10.1029/91JA02581.

Sonnerup, B. U., and M. Scheible (1998), Minimum and Maximum Variance Analysis, pp. 185-220, Int. Space Sci. Inst., Bern, Switzerland.

Spangler, S. R., J. A. Leckband, and I. H. Cairns (1997), Observations of the parametric decay instability of nonlinear magnetohydrodynamic waves, Phys. Plasmas, 4(3), 846-855, doi:10.1063/1.872183.

Strumik, M., V. Roytershteyn, H. Karimabadi, K. Stasiewicz, M. Grzesiak, and D. Przepiórka (2015), Identification of the dominant ULF wave mode and generation mechanism for obliquely propagating waves in the Earth's foreshock, Geophys. Res. Lett., 42, 5109-5116, doi:10.1002/2015GL064915.

Wang, X., C. Tu, L. Wang, J. He, and E. Marsch (2015), The upstream-propagating Alfvénic fluctuations with power law spectra in the upstream region of the Earth's bow shock, Geophys. Res. Lett., 42, 3654-3661, doi:10.1002/2015GL063893.

Wang, X. Y., and Y. Lin (2003), Generation of nonlinear Alfvén and magnetosonic waves by beam-plasma interaction, Phys. Plasmas, 10(9), 3528-3538, doi:10.1063/1.1599359.

Wang, X. Y., and Y. Lin (2006), Generation of filamentary structures by beam-plasma interaction, Phys. Plasmas, 13(5), 052102, doi:10.1063/1.2197797.

Wilkinson, W. P. (2003), The Earth's quasi-parallel bow shock: Review of observations and perspectives for Cluster, Planet. Space Sci., 51(11), 629-647, doi:10.1016/S0032-0633(03)00099-0, collisionless Shocks. 